\title{
Stability analysis of a continuous model of mutualism with delay dynamics
}

\author{
Joharjee, N.G.* Roberts, J.A. ${ }^{\dagger}$ \\ njoharji@gmail.com ～j.roberts@chester.ac.uk
}

January 12, 2016

\begin{abstract}
In this paper we introduce delay dynamics to a coupled system of ordinary differential equations which represent two interacting species exhibiting facultative mutualistic behaviour. The delays are representative of the beneficial effects of the indirect, interspecies interactions not being realised immediately. We show that the system with delay possesses a continuous solution, which is unique. Furthermore we show that, for suitably-behaved, positive initial functions that this unique solution is bounded and remains positive, i.e. both of the components representing the two species remain greater than zero. We show that the system has a positive equilibrium point and prove that this point is asymptotically stable for positive solutions and that this stability property is not conditional upon the delays.
\end{abstract}

\section{Model construction}

Although mutualism is widespread in nature, the theoretical, mathematical work in this area is not quite so prolific as the theoretical work related to predation and competition.

\footnotetext{
*Department of Mathematics, King Abdul Aziz University, Jeddah, KSA

${ }^{\dagger}$ Department of Mathematics, University of Chester, Thornton Science Park, Chester $\mathrm{CH} 24 \mathrm{NU}$
} 
Some early attempts at modelling mutualism involved taking a LotkaVolterra type model and changing negative coefficients to positive coefficients to represent mutualism rather than competition. Murray [6] (on pages 8384) describes this quite succinctly and presents the following model as one of the simplest attempts at turning a classical Lotka-Volterra model into a mutualism model:

$$
\begin{aligned}
& \frac{d x(t)}{d t}=a x(t)+b x(t) y(t), \\
& \frac{d y(t)}{d t}=c y(t)+d x(t) y(t),
\end{aligned}
$$

where $x(t)$ and $y(t)$ are the population sizes of the interacting species at time $t$, and $a, b, c, d$ are positive constants. The problem that can occur with this approach, as can be seen from this example, is that both $\frac{d x}{d t}$ and $\frac{d y}{d t}$ are positive, leading to unbounded growth.

Attempts to overcome such problems are discussed in a variety of texts, including [3] and [5] (pages 220-236). For these models to be more realistic some necessary conditions, such as weak or asymmetric interaction strengths, must be created to ensure the existence of a positive steady state (the stable coexistence). While these conditions on Lotka-Volterra models can prevent unbounded growth, they also convey that the mutualism has little effect on population dynamics. Due to these theoretical results, mutualism has been perceived as an interesting interaction, but one of less importance to ecological dynamics than predation or competition [3].

A model is described in [5] (and which also appears in [9]) where the mutualist decreases the density dependence in the per capita birth rate of the other species.

For the benefit of readers we describe how this model was constructed, noting that further discussions are available in [5] and [9].

Consider the existence of a species, whose population at a given moment in time is described by the continuous function $N_{1}(t)$. Suppose that the per capita birth rate decreases with density, so that it may be described by the relationship

$$
B_{1}(t)=b_{0}-b N_{1}(t)
$$

where both $b_{0}$ and $b$ are positive constants. We also impose the restriction that $B_{1}(t) \geq 0 \forall t \geq 0$. Similarly, we suppose that the per capita death rate increases with density, and once again the constants we introduce are 
positive, with a restriction that $D_{1}(t) \geq 0 \forall t \geq 0$ :

$$
D_{1}(t)=d_{0}+d N_{1}(t) \text {. }
$$

Therefore a growth rate of $\left(B_{1}(t)-D_{1}(t)\right) N_{1}(t)$ leads to the very familiar logistic differential equation

$$
\frac{d N_{1}(t)}{d t}=r N_{1}(t)-\frac{r}{K} N_{1}^{2}(t)
$$

where $r=b_{0}-d_{0}, K=\frac{b_{0}-d_{0}}{b+d}$. The introduction of $r$ and $K$ in this manner helpfully reduces the number of parameters.

We now introduce a facultative mutualist, i.e. a second species given by $N_{2}(t)$ where, although interactions between members of $N 1(t)$ and $N_{2}(t)$ provide a benefit to both populations, the interactions are not essential to the survival of either species. The benefit that $N_{2}(t)$ provides to $N_{1}(t)$ is realized through its ability to decrease the density dependence in the per capita birth rate of $N_{1}$ :

$$
B(t)=b_{0}-\frac{b N_{1}(t)}{1+\alpha_{12} N_{2}(t)} .
$$

The interaction therefore has no effect on the per capita death rate of $N_{1}(t)$.

Taking a symmetrical approach to constructing a differential equation for describing the $N_{2}(t)$ population yields the model discussed in [5] and [9]:

$$
\begin{aligned}
& \frac{d N_{1}(t)}{d t}=\left(r_{1}-\frac{b_{1} N_{1}(t)}{1+\alpha_{12} N_{2}(t)}-d_{1} N_{1}(t)\right) N_{1}(t), \\
& \frac{d N_{2}(t)}{d t}=\left(r_{2}-\frac{b_{2} N_{2}(t)}{1+\alpha_{21} N_{1}(t)}-d_{2} N_{2}(t)\right) N_{2}(t) .
\end{aligned}
$$

This model is fundamentally different to a model appearing in [2]. Both models avoid the unrealistic scenario of the orgy of mutual benefaction, which is present in many Lotka-Volterra based models of mutualism. However they have differing mutualistic mechanisms; they have a different biological meaning. The model appearing in [2] models a situation where each species benefits the other species directly. In the system (7), (8) each species decreases the density dependence in the per capita rate of the other species, which is an indirect benefaction. 
To be clear, in [4] direct interactions are deemed to be physical interactions between two species; for example, a plant relying on an animal or bird to transport their seeds to another location - thereby providing a food source for the animal (positive interaction) and dispersing the seed to increase the population level and geographic spread of the plant (again, positive interaction). In [10] it is noted that indirect interactions exist whereby, instead of two species interacting physically, a third species (possibly more) conveys the mutualistic effects. We describe two examples of indirect interactions in the next section.

There may be times when it is sensible to construct a system of three or more equations to model the interactions between the two mutualists and an 'intermediary' species. However, a lack of data or information of these intermediate interactions may make it difficult to formulate the necessary equations. In such a case, one could hypothesize that it may be beneficial to use a pair of equations where the construction takes account of indirect benefits and the absence, therefore of pairwise interactions.

\section{Examples of indirect interactions in nature}

In [7], where ants and their ecosystem are discussed in detail, it is stated that there are many occurrences of indirect mutualistic interactions. One such proposition in that text is the following scenario.

Consider a population of ants, which may be sub-divided into two subpopulations: dominant ants and sub-dominant ants. Some dominant ants may be more closely associated with homopterans producing honeydew (such as a myrmecophile species of aphid) whilst the sub-dominant ants may prefer to collect their nectar from a plant with extrafloral nectaries. The authors in [7] hypothesize that if the damage caused by the homopterans is low then the constant provision of sugar resources might lead to a positive, indirect interaction between homoptera and nectaries.

Another scenario described in [7] is one where ants attend a species providing honeydew or nectar and therefore provide protection to this species from its natural enemies. A second species, feeding in this vicinity may then be afforded the same protection, by virtue of proximity and could therefore be described as gaining an indirect benefit from the ants. Further, if this second species benefits the honeydew/nectar producing species then we can think of the ants benefiting indirectly from the second species due to the 
boost to their food source.

\section{$3 \quad$ Introducing delays to the model}

We now introduce delay dynamics into the model $(7),(8)$ and use an algebraic approach to extend the previously mentioned stability result to this new model.

Our approach is analogous to that used for the direct-benefaction model proposed in [2]. We introduce delays in (7) and (8) to reflect the possibility that mutualistic effects on the population are not realized instantaneously. Our new model has the form

$$
\begin{aligned}
& \frac{d N_{1}(t)}{d t}=\left(r_{1}-\frac{b_{1} N_{1}(t)}{1+\alpha_{12} N_{2}\left(t-\tau_{2}\right)}-d_{1} N_{1}(t)\right) N_{1}(t), \\
& \frac{d N_{2}(t)}{d t}=\left(r_{2}-\frac{b_{2} N_{2}(t)}{1+\alpha_{21} N_{1}\left(t-\tau_{1}\right)}-d_{2} N_{2}(t)\right) N_{2}(t) .
\end{aligned}
$$

Naturally, in order to obtain a specific solution, this model must be coupled with a pair of initial functions:

$$
\begin{aligned}
& N_{1}(t)=\varphi_{1}(t)>0 \text { for }-\tau \leq t \leq 0, \\
& N_{2}(t)=\varphi_{2}(t)>0 \text { for }-\tau \leq t \leq 0,
\end{aligned}
$$

where $\tau=\max \left\{\tau_{1}, \tau_{2}\right\}$ and each initial function is continuous over its domain and its range is the set of positive real numbers.

The introduction of the delays and their place within the model structure can be justified as follows. We have already established that it is not appropriate to consider pairwise interactions between the species and we are only interested in delays occurring in the indirect interactions between the species. Therefore this leaves only one place in each equation for introducing the delay term. If we think about the previously described examples of such interactions, it is easy to see how the benefits from indirect interactions can be delayed. For example, in our second example, it may take some time for the second species' impact on the environment to take hold; this environment being the same environment on which the honeydew/nectar producing species depends. 


\section{Establishing the existence of a unique so- lution}

Before we consider questions about stability we first address the issue of existence and uniqueness of a solution. We need to be sure that we have constructed a system of equations for which there is a unique solution. Furthermore, we show that such a solution is positive and bounded.

We may consider our system to be a special case of the following more general system of a pair of delay differential equations, with associated initial functions, written in vector form:

$$
\begin{gathered}
\mathbf{x}^{\prime}(t)=\mathbf{f}\left(t, \mathbf{x}(t), \mathbf{x}\left(t-s_{1}\right), \mathbf{x}\left(t-s_{2}\right)\right), \text { for } 0 \leq t \leq T \\
\mathbf{x}(t)=\boldsymbol{\theta}(t) \text { for }-\max \left\{s_{1}, s_{2}\right\} \leq t \leq 0 .
\end{gathered}
$$

Without loss of generality assume that $0 \leq s_{1}<s_{2}$. Consider the following existence-uniqueness theorem and associated proof, both adapted from [1]:

Theorem 4.1. Let $\mathbf{f}$ be continuous on $[0, T) \times D^{3}$, where $D^{3}$ is an open set in $\mathbb{R}^{3}$. Assume also that either the second order partial derivatives of each component of $\mathbf{f}$ exist and are continuous or that $\mathbf{f}$ satisfies a Lipschitz condition of the form

$$
\left\|\mathbf{f}\left(t, \xi_{(1)}, \xi_{(2)}\right)-\mathbf{f}\left(t, \tilde{\xi}_{(1)}, \xi_{(2)}\right)\right\| \leq K\left\|\xi_{(1)}-\tilde{\xi}_{(1)}\right\| .
$$

Additionally, assume that $\boldsymbol{\theta}$ is continuous on $\left[-\max \left\{s_{1}, s_{2}\right\}, 0\right]$. Then the system (13), (14) possesses a unique solution on any interval $\left[-\max \left\{s_{1}, s_{2}\right\}, \beta_{1}\right)$ where $0<\beta_{1} \leq \beta$.

Proof: We consider two cases: $s_{1}>0$ and $s_{1}=0$.

For $s_{1}>0$ we actually do not need our additional assumptions about the continuity of the partial derivatives or the existence of a Lipschitz condition. We have, for $0 \leq t \leq s_{1}$,

$$
\mathbf{x}^{\prime}(t)=\mathbf{f}\left(t, \boldsymbol{\theta}\left(t-s_{1}\right), \boldsymbol{\theta}\left(t-s_{2}\right)\right)
$$

and $\mathbf{x}(0)=\boldsymbol{\theta}(0)$. This uniquely determines

$$
\mathbf{x}(t)=\boldsymbol{\theta}(0)+\int_{0}^{t} \mathbf{f}\left(w, \boldsymbol{\theta}\left(w-s_{1}\right), \boldsymbol{\theta}\left(w-s_{2}\right)\right) d w
$$


for $0 \leq t \leq s_{1}$, provided $s_{1}<\beta$ and $\mathbf{x}(t)$ remains in $D$. Since we now know $\mathbf{x}(t)$ for $-s \leq t \leq s_{1}$, we can find $\mathbf{x}(t)$ for $s_{1} \leq t \leq 2 s_{1}$, provided $2 s_{1}<\beta$ and $\mathbf{x}(t)$ remains in $D$. This method of steps procedure continues until we reach $\beta$ or until $\mathbf{x}(t)$ reaches the boundary of $D$.

Now consider the $s_{1}=0$ case. For $0 \leq t \leq s_{2}$ we have

$$
\mathbf{x}^{\prime}(t)=\mathbf{f}\left(t, \mathbf{x}(t), \boldsymbol{\theta}\left(t-s_{2}\right)\right)
$$

with $\mathbf{x}(0)=\boldsymbol{\theta}(0)$. As such, each step in the method-of-steps construction process includes an ordinary differential equation and so, in order to demonstrate existence and uniqueness of a solution, we must introduce the kinds of conditions we see in existence-uniqueness theorems for ordinary differential equations; i.e. we require either the existence and continuity of the second order partial derivatives of each component of $\mathbf{f}$ or the aforementioned Lipschitz condition to be satisfied. More detailed discussions of such classical ordinary differential equation theory can be found in a variety of texts, including [1]. If $s_{2}<\beta$ we then consider $s_{2} \leq t \leq 2 s_{2}$, and so on.

We now state our own theorem which will require Theorem 4.1 to prove.

Theorem 4.2. The initial value problem consisting of the equations (9), (10), the initial functions (11), (12) and the additional conditions

$$
\begin{gathered}
r_{1}, r_{2}, b_{1}, b_{2}, d_{1}, d_{2} \in(0, \infty), \\
\alpha_{12}, \alpha_{21} \in[1, \infty),
\end{gathered}
$$

possesses a unique solution on the interval $[0, T)$ for some finite $T>0$. Furthermore, the components $N_{1}(t)$ and $N_{2}(t)$ of the solution are positive and bounded on the interval of existence.

Proof: It is clear that the system under consideration satisfies the conditions of Theorem 4.1 and so the existence of a unique solution is immediately obvious. For the convenience of readers who may be working through the detail, we present the second derivatives below: If we label the left hand sides of the equations (9) and (10) as the functions $f_{1}$ and $f_{2}$, respectively and consider each to be a function of $t, N_{1}(t), N_{2}(t), N_{1}\left(t-\tau_{1}\right), N_{2}\left(t-\tau_{2}\right)$ then the second derivatives will be as follows:

$$
\frac{\partial^{2} f_{1}}{\partial t^{2}}=\frac{\partial^{2} f_{2}}{\partial t^{2}}=\frac{\partial^{2} f_{1}}{\partial\left(N_{2}(t)\right)^{2}}=\frac{\partial^{2} f_{2}}{\partial\left(N_{1}(t)\right)^{2}}=\frac{\partial^{2} f_{1}}{\partial\left(N_{1}\left(t-\tau_{1}\right)\right)^{2}}=\frac{\partial^{2} f_{2}}{\partial\left(N_{2}\left(t-\tau_{2}\right)\right)^{2}}=0
$$




$$
\begin{gathered}
\frac{\partial^{2} f_{1}}{\partial\left(N_{1}(t)\right)^{2}}=r_{1}-\frac{2 b_{1}}{1+\alpha_{12} N_{2}\left(t-\tau_{2}\right)}, \\
\frac{\partial^{2} f_{2}}{\partial\left(N_{2}(t)\right)^{2}}=r_{2}-\frac{2 b_{2}}{1+\alpha_{21} N_{1}\left(t-\tau_{1}\right)}, \\
\frac{\partial^{2} f_{1}}{\partial\left(N_{2}\left(t-\tau_{2}\right)\right)^{2}}=\frac{-2 \alpha_{12}^{2} b_{1} N_{1}^{2}(t)}{\left(1+\alpha_{12} N_{2}\left(t-\tau_{2}\right)\right)^{3}}, \\
\frac{\partial^{2} f_{2}}{\partial\left(N_{1}\left(t-\tau_{1}\right)\right)^{2}}=\frac{-2 \alpha_{21}^{2} b_{2} N_{2}^{2}(t)}{\left(1+\alpha_{21} N_{1}\left(t-\tau_{1}\right)\right)^{3}} .
\end{gathered}
$$

We require nonzero denominators in these derivatives. This is guaranteed by the facts that our initial functions are positive and our analysis below which demonstrates that $N_{1}(t), N_{2}(t)$ remain positive, and indeed bounded, for positive values of $t$.

We show positivity and boundedness for $N_{1}(t)$ and note that an identical argument will establish the same property for $N_{2}(t)$. Firstly, we see that

$$
\frac{d N_{1}(t)}{d t} \geq\left(r_{1}-\left(b_{1}+d_{1}\right) N_{1}(t)\right) N_{1}(t)
$$

Thus $N_{1}(t)$ will only grow if

$$
\frac{r_{1}}{b_{1}+d_{1}}>N_{1}(t)
$$

In other words, should $N_{1}(t)$ grow beyond $\frac{r_{1}}{b_{1}+d_{1}}$ then it will cease to grow any larger. Secondly, we can also see that

$$
\frac{d N_{1}(t)}{d t} \leq r_{1} N_{1}(t)-d_{1} N_{1}^{2}(t)
$$

Thus $N_{1}(t)$ will only decrease if

$$
N_{1}(t)>\frac{r_{1}}{d_{1}}
$$

That is, should $N_{1}(t)$ fall below this non-zero threshold it will cease to decrease further. Thus we conclude that the populations $N_{1}(t)$ and, via a similar argument $N_{2}(t)$ are both bounded and permanent, as required. 


\section{Asymptotic stability of the positive equi- librium point}

It is well understood that the system (7), (8) has an asymptotically stable nontrivial equilibrium for $r_{1}, r_{2}, b_{1}, b_{2}, d_{1}, d_{2} \in(0, \infty), \alpha_{12}, \alpha_{21} \in[1, \infty)$. The method of isoclines is discussed (for example in [5] and [9]) as a way to demonstrate this. We now prove a stability result for our model with delays. Another approach which yields success with some models is that of applying Lyapunov functions or functionals; the text by Shaikhet [8], in particular Chapter 10, demonstrates this approach with a number of ecological models. Our approach here is inspired by some of Gopalsamy's techniques in [2]. In order to prove our stability result we need to prove the following inequality is true.

Lemma 5.1. If $r_{1}, r_{2}, b_{1}, b_{2}, d_{1}, d_{2} \in(0, \infty), \alpha_{12}, \alpha_{21} \in[1, \infty)$ then

$$
\frac{\alpha_{12} \alpha_{21} b_{1} b_{2}\left(N_{1}^{*} N_{2}^{*}\right)^{2}}{\left(1+\alpha_{12} N_{2}^{*}\right)^{2}\left(1+\alpha_{21} N_{1}^{*}\right)^{2}}<r_{1} r_{2},
$$

where $\left(N_{1}^{*}, N_{2}^{*}\right)$ is the positive steady state of the system (9),(10),(11),(12).

Proof: Note first that

$$
N_{1}^{*} N_{2}^{*}=\frac{r_{1} r_{2}\left(1+\alpha_{12} N_{2}^{*}\right)\left(1+\alpha_{21} N_{1}^{*}\right)}{\left(b_{1}+d_{1}\left(1+\alpha_{12} N_{2}^{*}\right)\right)\left(b_{2}+d_{2}\left(1+\alpha_{21} N_{1}^{*}\right)\right)}
$$

and

$$
r_{1} r_{2}=\frac{\left(b_{1}+d_{1}\left(1+\alpha_{12} N_{2}^{*}\right)\right)\left(b_{2}+d_{2}\left(1+\alpha_{21} N_{1}^{*}\right)\right) N_{1}^{*} N_{2}^{*}}{\left(1+\alpha_{12} N_{2}^{*}\right)\left(1+\alpha_{21} N_{1}^{*}\right)}
$$

So

$$
\begin{aligned}
\frac{\alpha_{12} \alpha_{21} b_{1} b_{2}\left(N_{1}^{*} N_{2}^{*}\right)^{2}}{\left(1+\alpha_{12} N_{2}^{*}\right)^{2}\left(1+\alpha_{21} N_{1}^{*}\right)^{2}} & <\frac{\alpha_{12} \alpha_{21} N_{1}^{*} N_{2}^{*} r_{1} r_{2}}{\left(1+\alpha_{12} N_{2}^{*}\right)\left(1+\alpha_{21} N_{1}^{*}\right)} \\
& <r_{1} r_{2},
\end{aligned}
$$

as required.

We now state and prove our stability theorem for this delay model.

Theorem 5.1. Assume that $r_{1}, r_{2}, b_{1}, b_{2}, d_{1}, d_{2} \in(0, \infty), \alpha_{12}, \alpha_{21} \in[1, \infty)$. Then the positive steady state $E=\left(N_{1}^{*}, N_{2}^{*}\right)$ of the delay differential system (9),(10) with initial functions (11),(12) is asymptotically stable within the positive quadrant. 
Proof: First, it is rather straightforward to solve $\frac{d N_{1}(t)}{d t}=\frac{d N_{2}(t)}{d t}=0$ in order to identify the non-trivial equilibrium point (i.e. that equilibrium point which is in the positive quadrant) as

$$
E=\left(N_{1}^{*}, N_{2}^{*}\right)=\left(\frac{r_{1}\left(1+\alpha_{12} N_{2}\right)}{b_{1}+d_{1}\left(1+\alpha_{12} N_{2}\right)}, \frac{r_{2}\left(1+\alpha_{21} N_{1}\right)}{b_{2}+d_{2}\left(1+\alpha_{21} N_{1}\right)}\right) .
$$

We also note that this is identical to the non-trivial equilibrium point for the system without delay.

The Jacobian matrix of the system, evaluated at this equilibrium point is

$$
J_{E}=\left(\begin{array}{ll}
-r_{1} & \frac{\alpha_{12} b_{1}\left(N_{1}^{*}\right)^{2}}{\left(1+\alpha_{12} N_{2}^{*}\right)^{2}} \exp \left(-\lambda \tau_{2}\right) \\
\frac{\alpha_{21} b_{2}\left(N_{2}^{*}\right)^{2}}{\left(1+\alpha_{21} N_{1}^{*}\right)^{2}} \exp \left(-\lambda \tau_{1}\right) & -r_{2}
\end{array}\right) .
$$

Using $\operatorname{det}\left(J_{E}-\lambda I\right)=0$ we obtain the following characteristic equation:

$$
\lambda^{2}+A \lambda+B-C \exp (-\lambda \tau)=0
$$

where

$$
\begin{aligned}
A & =r_{1}+r_{2}, \\
B & =r_{1} r_{2} \\
C & =\frac{b_{1} b_{2} \alpha_{12} \alpha_{21}\left(N_{1}^{*} N_{2}^{*}\right)^{2}}{\left(1+\alpha_{12} N_{2}^{*}\right)^{2}\left(1+\alpha_{21} N_{1}^{*}\right)^{2}}, \\
\tau & =\tau_{1}+\tau_{2} .
\end{aligned}
$$

Asymptotic stability of the point $E$ is assured if the roots of the characteristic equation have negative real part. Substituting $\lambda=\alpha+i \beta$ into (26) and separating the real and imaginary parts yields the following two equations:

$$
\begin{aligned}
\left(\alpha^{2}-\beta^{2}\right)+(\alpha A+B) & =C \exp (-\alpha \tau) \cos (\beta \tau) \\
2 \alpha \beta+\beta A & =-C \exp (-\alpha \tau) \sin (\beta \tau) .
\end{aligned}
$$

Squaring and adding (31), (32) yields the single equation

$$
\begin{gathered}
\left(\alpha^{2}+\beta^{2}\right)^{2}+\alpha^{2} A^{2}+\beta^{2}\left(2 \alpha A+A^{2}-2 B\right)+ \\
2 \alpha\left(\alpha^{2} A+\alpha B+A B\right)+B^{2}\left(1-\frac{C^{2}}{B^{2}} \exp (-2 \alpha \tau)\right)=0
\end{gathered}
$$


Using Lemma 5.1 we have the additional information that

$$
0<C<B \text {. }
$$

It is also clear that $A B>0$ and $A^{2}-2 B>0$. If, by way of contradiction, we assume $\alpha \geq 0$ then (33) is only satisfied if $\alpha=\beta=0$ and $B=C$. This contradicts (34) and so we conclude that every root of the characteristic equation (26) has a negative real part. This concludes the proof.

We note that the asymptotic stability is not conditional on the delay and it is immediately obvious that asymptotic stability of the underlying ordinary differential system proposed in [5] and [9] can be thought of as a special case of this result, thus giving us an algebraic proof for the non-delay case as an alternative to the method of isoclines.

\section{Acknowledgements}

The authors are grateful to King Abdul Aziz University, Jeddah for funding Dr Joharjee's visit to the University of Chester, England in order to facilitate this collaboration.

\section{References}

[1] Driver, R.D., 1978. Introduction to Ordinary Differential Equations, Harper and Row Publishers.

[2] Gopalsamy, K., 1992. Stability and Oscillations in Delay Differential Equations of Population Dynamics, Kluwer Academic Publishers.

[3] Holland, N.J., 2012. Population Dynamics of Mutualism, Nature Education Knowledge 3(10):2.

[4] Holt, R.D., Lawton, J.H., 1994. The ecological consequences of shared natural enemies, Annual Review of Ecology and Systematics 25, 495520 .

[5] Kot, M., 2001. Elements of Mathematical Ecology, Cambridge University Press.

[6] Murray, J.D., Mathematical Biology (Second Ed.), Springer, 1993. 
[7] Stadler, B., Dixon, T., 2008. Mutualism: Ants and their insect partners, Cambridge University Press.

[8] Shaikhet, L., 2013. Lyapunov Functionals and Stability of Stochastic Functional Differential Equations, Springer.

[9] Wolin, C.L., Lawlor, L.R., 1984. Models of facultative mutualism:density effects, American Naturalist 124, 843-862.

[10] Wootton, J.T., 1994. The nature and consequences of indirect effects in ecological communities, Annual Review of Ecology and Systematics 25, 443-466. 